\title{
Considerations for Application of SOBI to Order Tracking
}

\section{Scot I. McNeill}

Stress Engineering Services, Houston, USA.

Email: scot.mcneill@stress.com

Received November $22^{\text {nd }}, 2010$; revised December $8^{\text {th }}, 2011$; accepted December $9^{\text {th }}, 2011$

\begin{abstract}
Current methods of order tracking, such as synchronous resampling, Gabor filtering, and Vold-Kalman filtering have undesirable traits. Each method has two or more of the following deficiencies: requires measurement or estimate of rotational speed over time, failure to isolate the contribution of crossing orders in the vicinity of the crossing time, large computational expense, end effects. In this work a new approach to the order tracking problem is taken. The Second Order Blind Identification (SOBI) algorithm is applied to synthesized data. The technique is shown to be very successful at isolating crossing orders and circumvents all of the above deficiencies. The method has its own restrictions: multiple sensors are required and sensors must be mounted on a structure that responds quasi-statically to excitation of the rotational system.
\end{abstract}

Keywords: Blind Source Separation (BSS), Order Tracking, Second Order Blind Identification (SOBI), Synchronous Resampling, Vold-Kalman Filter, Gabor Filter

\section{Introduction}

Order tracking methods attempt to isolate the periodic components (orders) in rotating machinery vibration. Most often, Root Mean Square (RMS) vibration levels are estimated for each order as a function of rotational speed. Current methods of order tracking, such as synchronous resampling [1], Gabor filtering [2], and VoldKalman filtering [3] have some undesirable qualities. Synchronous resampling and Gabor filtering methods are unable to isolate the contribution of crossing orders in the vicinity of the crossing time (time at which the orders have the same frequency). Vold-Kalman filtering is able to resolve crossing orders, but only at great computational expense. Vold-Kalman filtering also exhibits end effects, where the beginning and end of the order time traces are distorted due to slow rise-time of the filter. All of the methods mentioned require measurement or construction of the rotational speed over time.

Blind Source Separation (BSS) techniques have been developed in the recent literature to decompose measured signals into fundamental components. One such technique, the Second Order Blind Identification (SOBI) algorithm [4] has been successfully adapted to estimate modal responses from measured vibration data [5]. Since system orders are essentially amplitude and frequency modulated sinusoids, SOBI is effective at isolating the system orders for the same reasons that it is effective at estimating modal responses.

In this work, the SOBI algorithm is considered for order tracking. The advantages and limitations are discussed. The technique is used to isolate the orders from synthetic data. The next section discusses the application of BSS techniques to the order tracking problem. The numerical example is provided in Section 3. Observations are summarized in Section 4.

\section{BSS for Order Tracking}

BSS attempts to find special source components, $s_{j}(t)$, embedded in measured data $x_{i}(t)$. The technique proposed in this work for order identification assumes that the measured data is a linear mixture (as opposed to convolutive) of the components. Suppose that there are $m$ channels of measured data and $n$ components. Making the time dependence implicit, the relation between the components and the measured data can be written as

$$
\mathbf{x}^{(\mathrm{mx} 1)}=\mathbf{A}^{(\mathrm{m} \times \mathrm{n})} \mathbf{s}^{(\mathrm{n} \times 1)},
$$

where $\mathbf{A}$ is the (constant) mixing matrix and the dimensions have been placed in the superscript. All quantities are real valued. The objective of BSS is to simultane- 
ously estimate the mixing matrix, $\mathbf{A}$, and the vector of components, $\mathbf{s}(t)$, from the observed data, $\mathbf{x}(t)$. Due to the number of variables involved, this task requires a characterization of the source components, $\mathbf{s}(t)$. Many BSS techniques use second order statistical information (e.g. variance) to describe the components, while ICA typically uses higher order statistics (e.g. kurtosis). It is appropriate to consider the inverse relationship of (1),

$$
\mathbf{s}^{(\mathrm{n} \times 1)}=\mathbf{W}^{(\mathrm{n} \times \mathrm{m})} \mathbf{x}^{(\mathrm{m} \times 1)} \text {. }
$$

The de-mixing matrix, $\mathbf{W}$, is the (generalized, if necessary) inverse of the mixing matrix, $\mathbf{A}$. The task is now to estimate $\mathbf{W}$ and $\mathbf{s}(t)$. Note that in order to estimate the $n$ components, it is necessary to have enough independent observations. This requires $m \geq n$ and the rank of $\mathbf{A}$ is $n$. It can be seen that the number of components, $n$, must be deduced from the observed data $\mathbf{x}(t)$. This can be accomplished by plotting the time-frequency distribution computed using short-time Fourier transform, for example.

Because the inverse of the mixing matrix, $\mathbf{W}$, and the components, $\mathbf{s}(t)$, must be estimated simultaneously, any scalar multiplier of one of the components $s_{j}(t)$ could be canceled by dividing the corresponding column $\mathbf{a}_{j}$ by the same scalar. This leads to some ambiguities. First, the variances of the independent components cannot be determined. This means that the amplitude and sign of each component $s_{j}(t)$ are unknown. A natural way to fix the amplitude of each component is to set the variance equal to one: $E\left\{s_{j}^{2}(\mathrm{t})\right\}=1$. Note that the sign is still ambiguous. Second, the order of importance of the components is unknown. This is in contrast to the familiar Principal Component Analysis (PCA), where the principal components are ordered by their variance.

The potential application of BSS on the order tracking problem is now discussed. Suppose a system responds quasi-statically to excitation from one or more rotating components. The vector of structural responses, $\mathbf{x}(t)$, are related to the exciting forces, $\mathbf{f}(t)$, by multiplication of flexibility matrix, $\mathbf{G}$,

$$
\mathbf{x}=\mathbf{G f} .
$$

Note that the flexibility matrix is the inverse of the stiffness matrix, $\mathbf{G}=\mathbf{K}^{-1}$. The force vector can be expressed as a linear combination of force components from each system order, $\mathbf{p}(t)$,

$$
\mathbf{f}=\mathbf{C p} \text {. }
$$

Substituting (4) into (3), we arrive at,

$$
\mathbf{x}=\boldsymbol{\Phi} \mathbf{p}, \text { where } \boldsymbol{\Phi}=\mathbf{G C} .
$$

One might consider using BSS to estimate both the (inverse) weighting matrix, $\boldsymbol{\Phi}$, and the order forces,

$$
\mathbf{p}=\boldsymbol{\Phi}^{-1} \mathbf{x} \text {. }
$$

Observe that this is consistent with the mixing model, Equations (1) and (2).

The SOBI algorithm is ideally suited for estimating the order forces, $\mathbf{p}(t)$, due to the correlation structure of the harmonic components comprising the elements of $\mathbf{p}(t)$. SOBI finds components that are uncorrelated with one another, irrespective of a small time shift between the two signals. Harmonically related sinusoids are orthogonal and thus possess this property. Details of the SOBI algorithm may be found in references [4,5] as well as many other references.

Order tracking using the SOBI algorithm essentially estimates the orders as a linear transform of the measured data. As such, it does not suffer from the drawbacks of the traditional methods discussed in Section 1. However it carries its own restrictions. The number of independent measurements, $m$, must be at least as large as the number of orders to estimate, $n$. In addition, the sensors must be mounted on a structure that responds quasi-statically to the forces from the rotational component. It should also be noted that the resulting orders are normalized to unit variance. The first restriction can be alleviated to some extent by filtering the data into bands using a band-pass filter. For best performance, the filter may be adaptive, tracking the time varying frequency. The second restriction can limit the application of the method. It is important to note that resonances of the rotating component, such as shaft criticals, can be handled by the method. However dynamics of the structure on which measurements are taken is not accounted for in the theoretical development. One may consider application of a BSS method that assumes a convolutive mixture of the source components. The third restriction does not present much of a problem since the strength of order $j$ in measurement $i$ is given by the estimated mixing matrix element $a_{i j}$.

\section{Application of SOBI to Synthesized Data}

In order to investigate the effectiveness of the SOBI algorithm in separating system orders, a data set was synthesized from six amplitude and frequency modulated sinusoids. The data set consists of a sinusoid with upsweeping frequency along with two harmonics, and a down sweeping frequency with two harmonics. Amplitude modulation was introduced by multiplying by an envelope sinusoid with a period slightly longer than the entire data set and random phase angle. The sinusoids were then mixed together using a $8 \times 6$ random mixing matrix to generate eight synthetic measurements. Uncorrelated Gaussian random noise with RMS equal to $5 \%$ of the modulated sinusoid RMS was added to the synthetic measurements. Due to the combination of up and down sweeping signals, several order crossings are present in the data. 
An example time series and spectrogram of one of the synthetic measurements is shown in Figure 1 and Figure $\mathbf{2}$, respectively. It can be seen that the data is composed of a mixture of the six orders. The many order crossings are also evident. Figure 3 shows the original orders and those estimated using SOBI. The same plot zoomed in with markers added to the data points can be seen in Figure 4. The estimates essentially overlay the original orders. However, due to the added noise, there is a small error in the estimates. The error sequence can be defined as the difference between the original order and the estimate,

$$
\mathbf{e}(t)=\hat{\mathbf{p}}(t)-\mathbf{p}(t) \text {. }
$$

The maximum RMS error is only $4.5 \%$ of the order RMS. The small error is due to the $5 \%$ RMS noise that was added to the synthetic measurements. Figure 5 shows the spectrogram of the estimated orders. The orders are clearly isolated quite well.

The traditional RMS vs. angular rate plots can be created using the resulting time series of the estimated orders. Instantaneous frequency, in Rotations Per Minute (RPM), can be computed from the fundamental estimated orders using the spectrogram, zero-crossing frequency, or Hilbert transform angles. The running RMS can easily be calculated from each estimated order and plotted vs. RPM. Figure 6 shows such a plot for the estimated orders. The fundamental up-sweeping RPM was used for orders 1-3 and the fundamental down-sweeping RPM was used for orders 4-6.

\section{Summary}

The common order tracking methods have several drawbacks associated with their use: methods require measurement or estimate of rotational speed over time, some methods fail to isolate the contribution of crossing orders in the vicinity of the crossing time, some methods require large computational expense, some methods are prone to end effects. In this work the SOBI algorithm was applied to the order tracking problem. The method

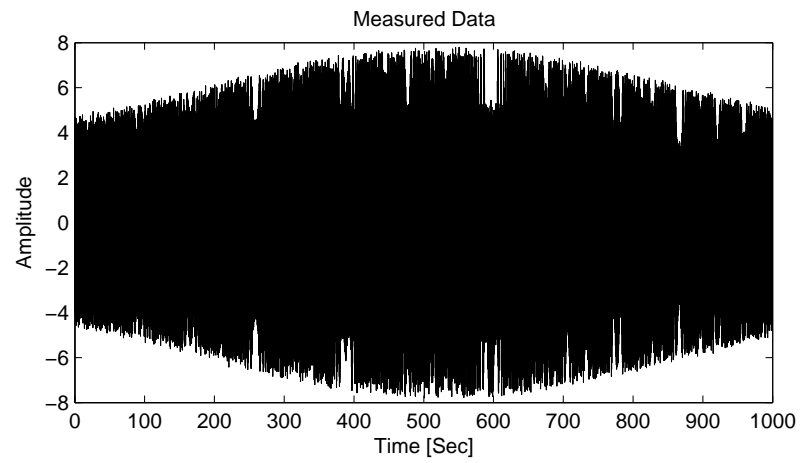

Figure 1. Example synthetic measured data (time series).

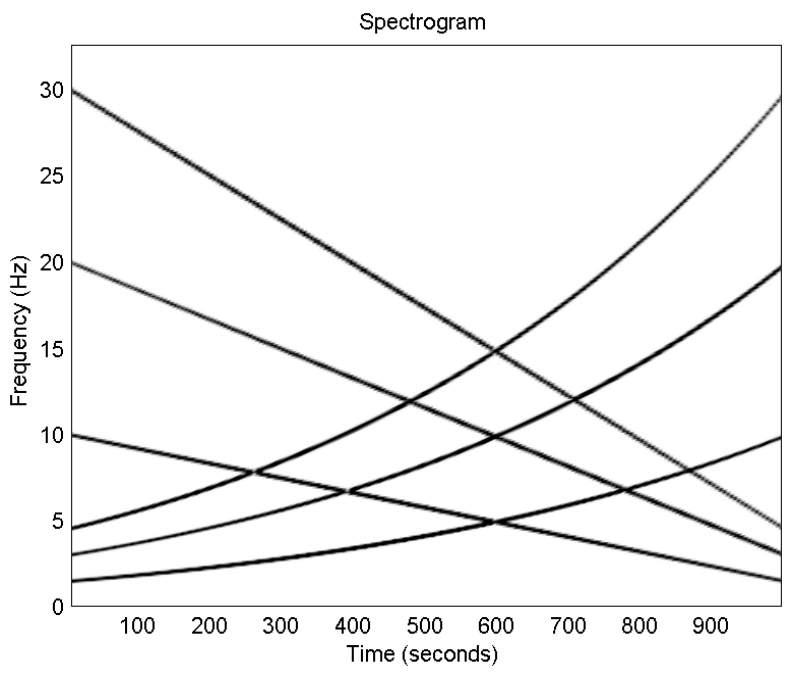

Figure 2. Example synthetic measured data (spectrogram).
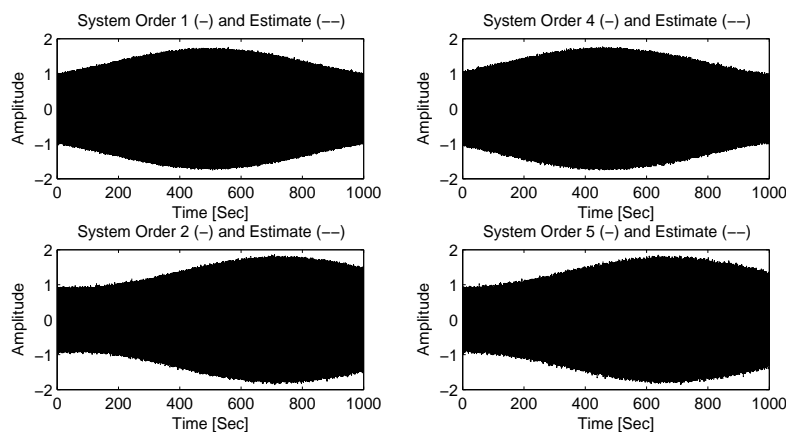

System Order $3(-)$ and Estimate (--)
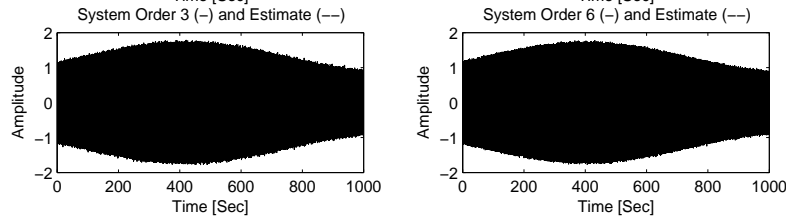

Figure 3. Original and estimated orders.
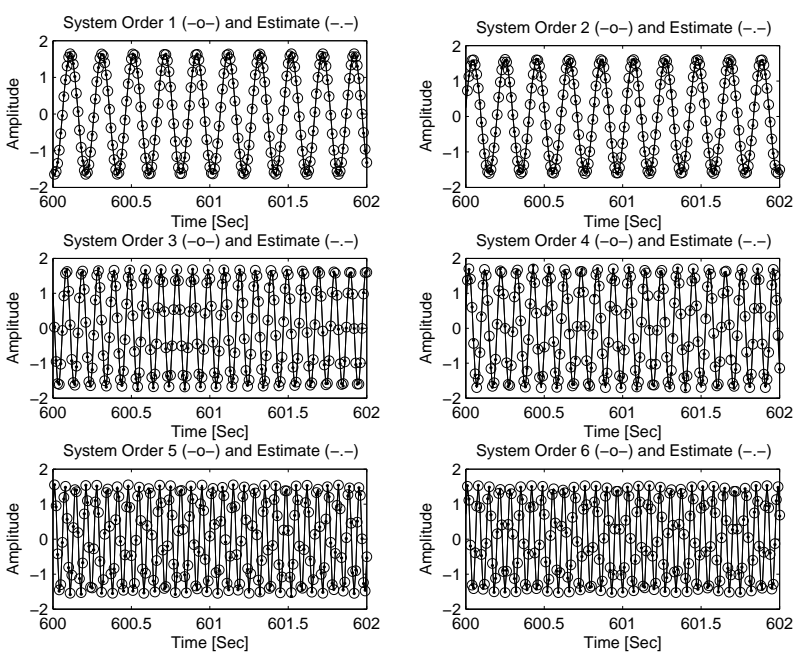

Figure 4. Original and estimated orders (zoomed in). 

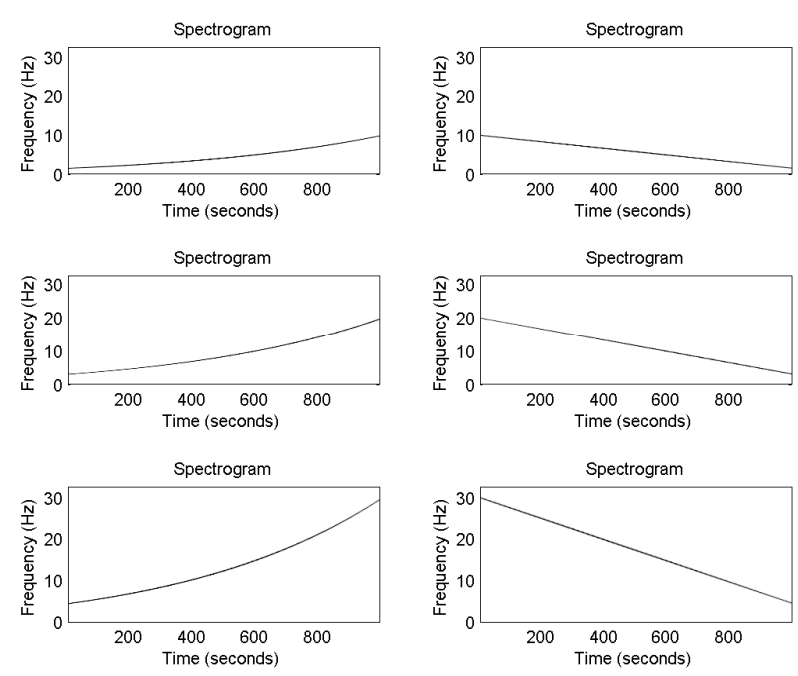

Figure 5. Estimated orders (spectrogram).
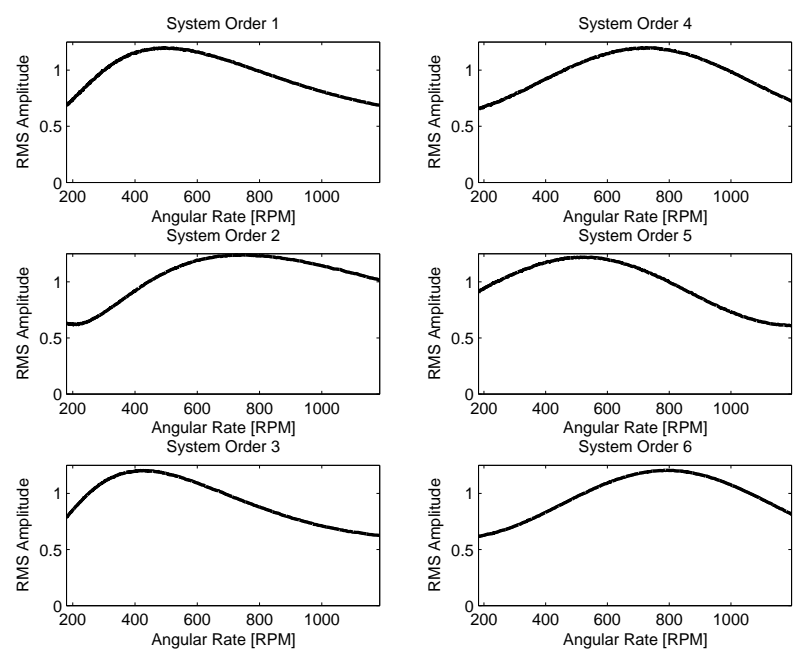

Figure 6. RMS of estimated orders. circumvents the drawbacks of the techniques currently used for order tracking. However the method has its own restrictions, associated with the SOBI algorithm: multiple sensors are required and sensors must be mounted on a structure that responds quasi-statically to excitation of the rotational system.

Performance of the method was examined by application to a synthesized data set consisting of three upsweeping components and three down-sweeping components with amplitude modulation. Uncorrelated Gaussian noise with 5\% RMS was added. The algorithm was extremely effective at isolating the six orders with no prior knowledge of the frequency content. No end effects or errors in the vicinity of the order crossings were evident. Maximum RMS error between the original orders and the estimates was $4.5 \%$, which is less than the additive noise.

\section{REFERENCES}

[1] A. Brandt, T. Lago, K. Ahlin and J. Tuma, "Main Principles and Limitations of Current Order Tracking Methods," Sound and Vibration Magazine, Vol. 39, No. 3, 2005, pp. 19-22.

[2] S. Qian, "Gabor Expansion for Order Tracking," Sound and Vibration Magazine, Vol. 37, No. 6, 2003, pp. 18-22.

[3] H. Vold, J. Leuridan, "High Resolution Order Tracking at Extreme Slew Rates, using Kalman Tracking Filter", Society of Automotive Engineers paper 931288, May 1993.

[4] A. Belouchrani, K. Abed-Meraim, J.-F. Cardoso and E. Moulines, "A Blind Source Separation Technique using Second-Order Statistics," IEEE Transactions on Signal Processing, Vol. 45, No. 2, 1997, pp. 434-444. doi:10.1109/78.554307

[5] S. I. McNeill and D. C. Zimmerman, "A Framework for Blind Modal Identification using Joint Approximate Diagonalization," Mechanical Systems and Signal Processing, Vol. 22, No. 7, 2008, pp. 1526-1548. doi:10.1016 j.ymssp.2008.01.010 